\title{
Progress and Plans for a US Laser System for the LISA Mission
}

Kenji Numata, Anthony Yu, Hua Jiao, Scott Merritt, Frankie Micalizzi, Molly Fahey, Jordan Camp, Michael Krainak

NASA Goddard Space Flight Center

9 October 2018

International Conference on Space Optics

Chania, Greece 


\section{Contents}

○ 1. Introduction

- LISA requirements

- Redundancy

$\circ$ 2. NASA laser architecture

- Master Oscillator Power Amplifier (MOPA)

- Laser head (LH) layout

$\circ$ 3. NASA laser activities

- Master oscillator (MO)

- Power amplifier (PA)

○ 4. Summary 


\section{Introduction}

○ High level LISA laser requirements

- Wavelength \& power

- Wavelength:1064.49nm, >2W on optical bench at EoL,

- Size \& mass

- $200 \times 200 \times 200 \mathrm{~mm}$ (incl. electronics), $10 \mathrm{~kg}$ per laser head (LH)

- Lifetime

- >16 years (including ground testing, cruise, normal and extended science ops)

- Noise requirements

- Frequency, intensity, RF phase noise

- Other requirements

- Dissipated power $<50 \mathrm{~W}$

- OP temperature $20 \pm 10^{\circ} \mathrm{C}$

- NOP temperature $-20^{\circ} \mathrm{C}$ to $+50^{\circ} \mathrm{C}$

- Few mW pick-off for Laser Pre-stabilization System (LPS)

- Linear polarization 


\section{Redundancy}

- Each payload has two laser system (LS) assemblies

- Each laser system comprises a Laser Optical Module (LOM) and Laser Electrical Module (LEM).

- Each laser system (LS) contains two Laser Heads (LH)

-4 per S/C, 12 in the constellation

- Cold redundancy

- One Laser Pre-stabilization System (LPS) per S/C

- Redundancy at constellation level (3 in the constellation)

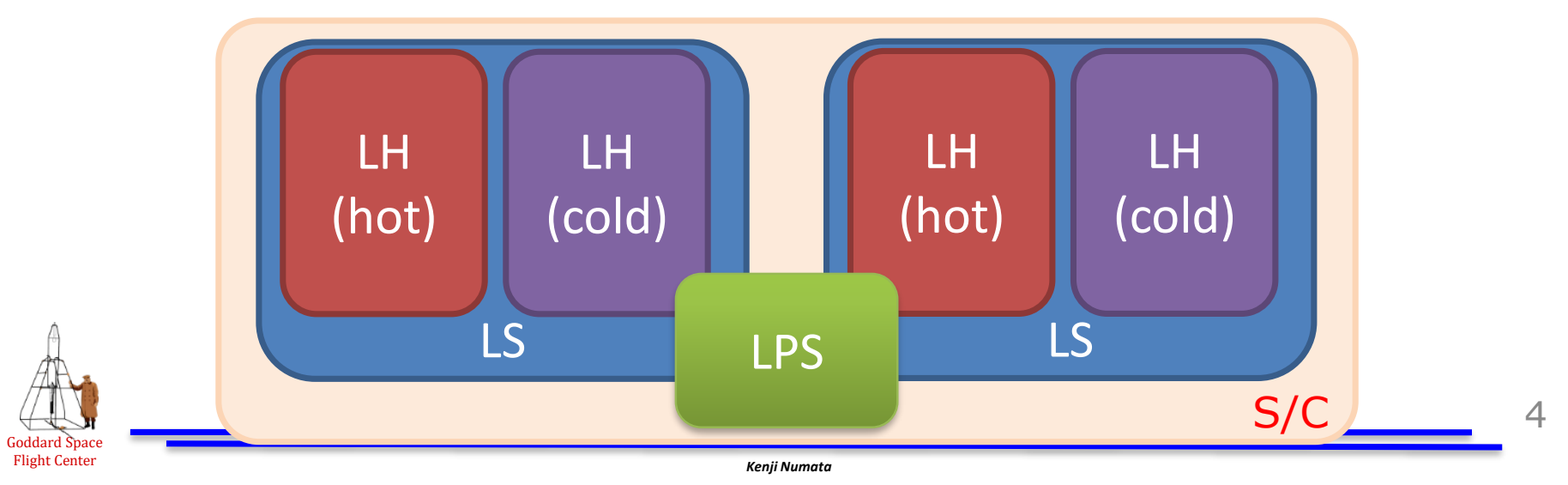




\section{NASA Laser Architecture}

○ Master oscillator power amplifier (MOPA)

- A MOPA architecture separates the problem

- Low noise, high power, and $\mathrm{GHz}$ phase modulation

- The MO dominates the frequency noise.

- Low power, low noise MO, followed by a fiber-coupled phase modulator

- The PA dominates the RIN and RF phase noise.

- Low noise power amplifier for power scaling of the modulated MO output.

\begin{tabular}{|c|c|c|}
\hline & $\begin{array}{c}\text { Master oscillator } \\
(\text { MO) }\end{array}$ & $\begin{array}{c}\text { Power amplifier } \\
\text { (PA) }\end{array}$ \\
\hline GSFC in-house & micro-NPRO (AVO) & $\begin{array}{c}\text { LMA Yb fiber amp. (LGS) } \\
\text { Nd:YAG crystal fiber }\end{array}$ \\
\hline
\end{tabular}

External vendor

Conventional NPRO

(Coherent)
Yb fiber amps

(Fibertek, Advalue, vendor \#3) 


\section{Preliminary laser head layout}

- Concept

- Full redundant MO

- 2 MOs per LH

- 2 pump LDs per MO

- Full redundant phase modulator (PM)

- 2 PMs per LH

- Redundant pump diodes for PA

- 2 pump LDs per PA

- Extending pump LD lifetime

- >50\% de-rating for pump LDs

- Simultaneous use of multiple LDs

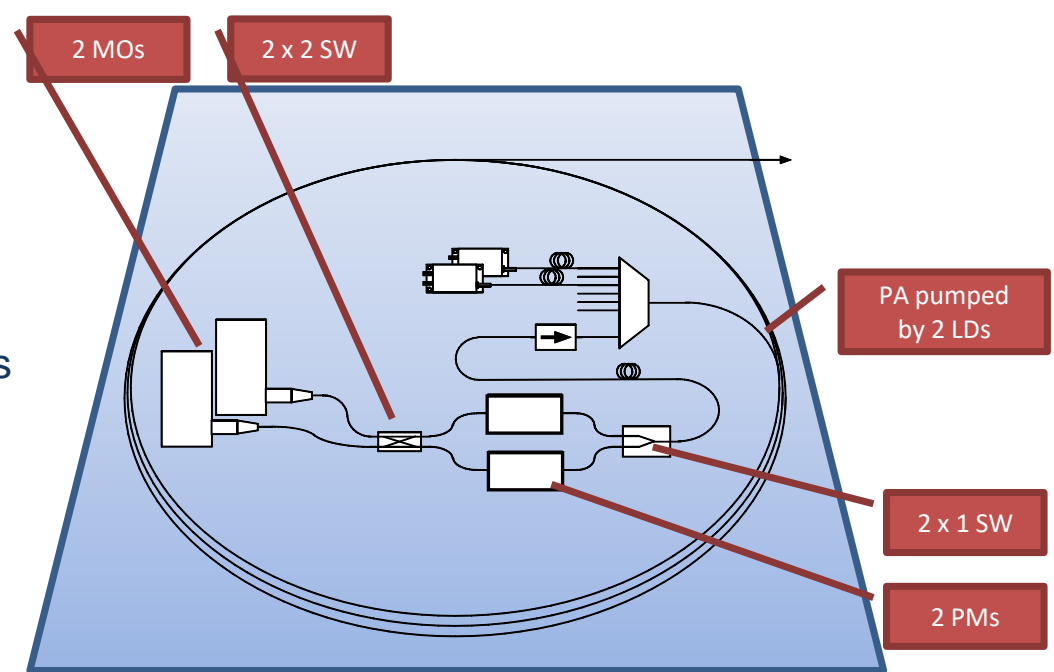

LOM of LH $(200 \mathrm{~mm} \times 200 \mathrm{~mm}$ area $)$ 


\section{Laser Optical Module (LOM)}

○ Not including redundancy within LH
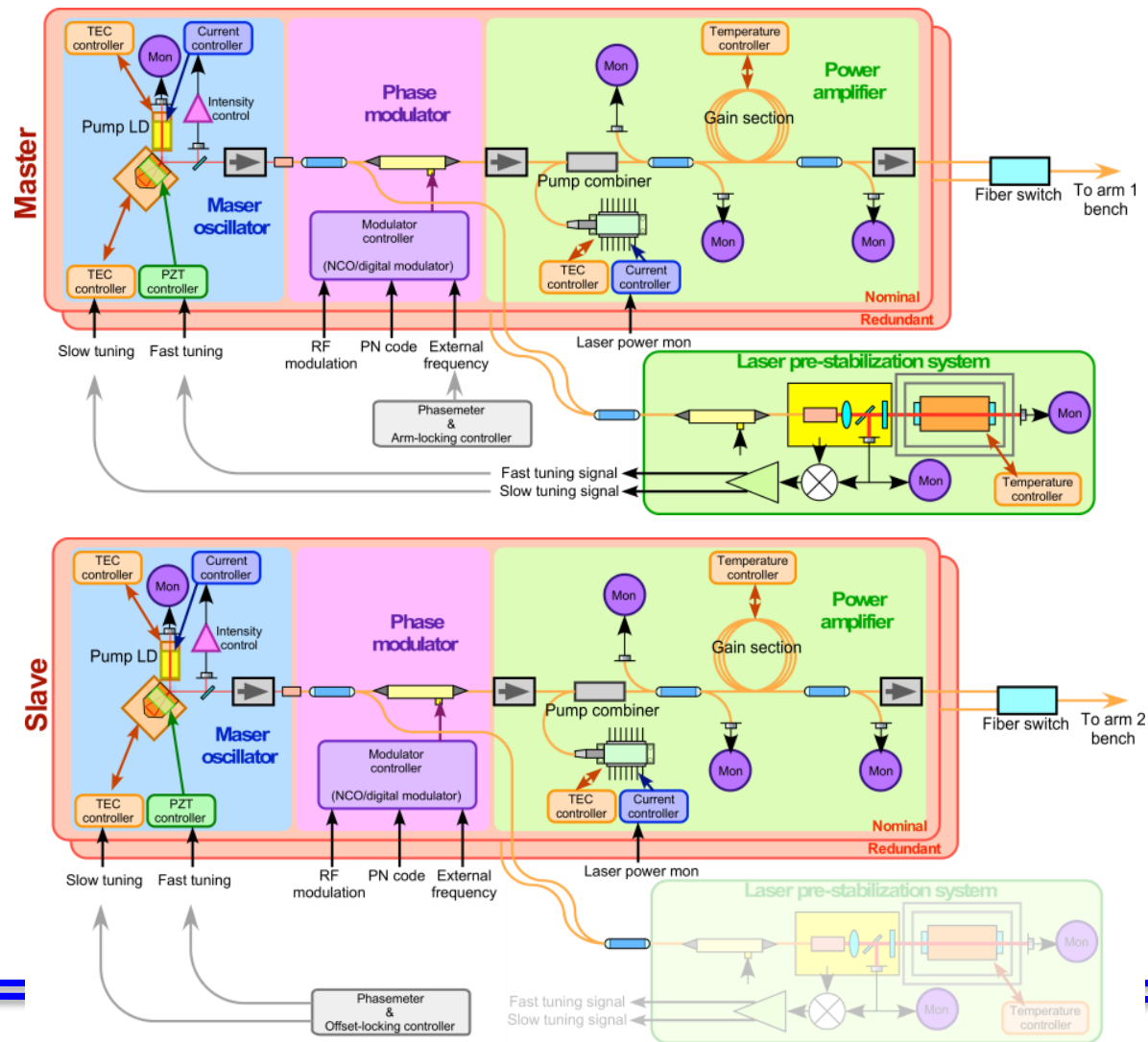


\section{Laser Electronics Module (LEM)}
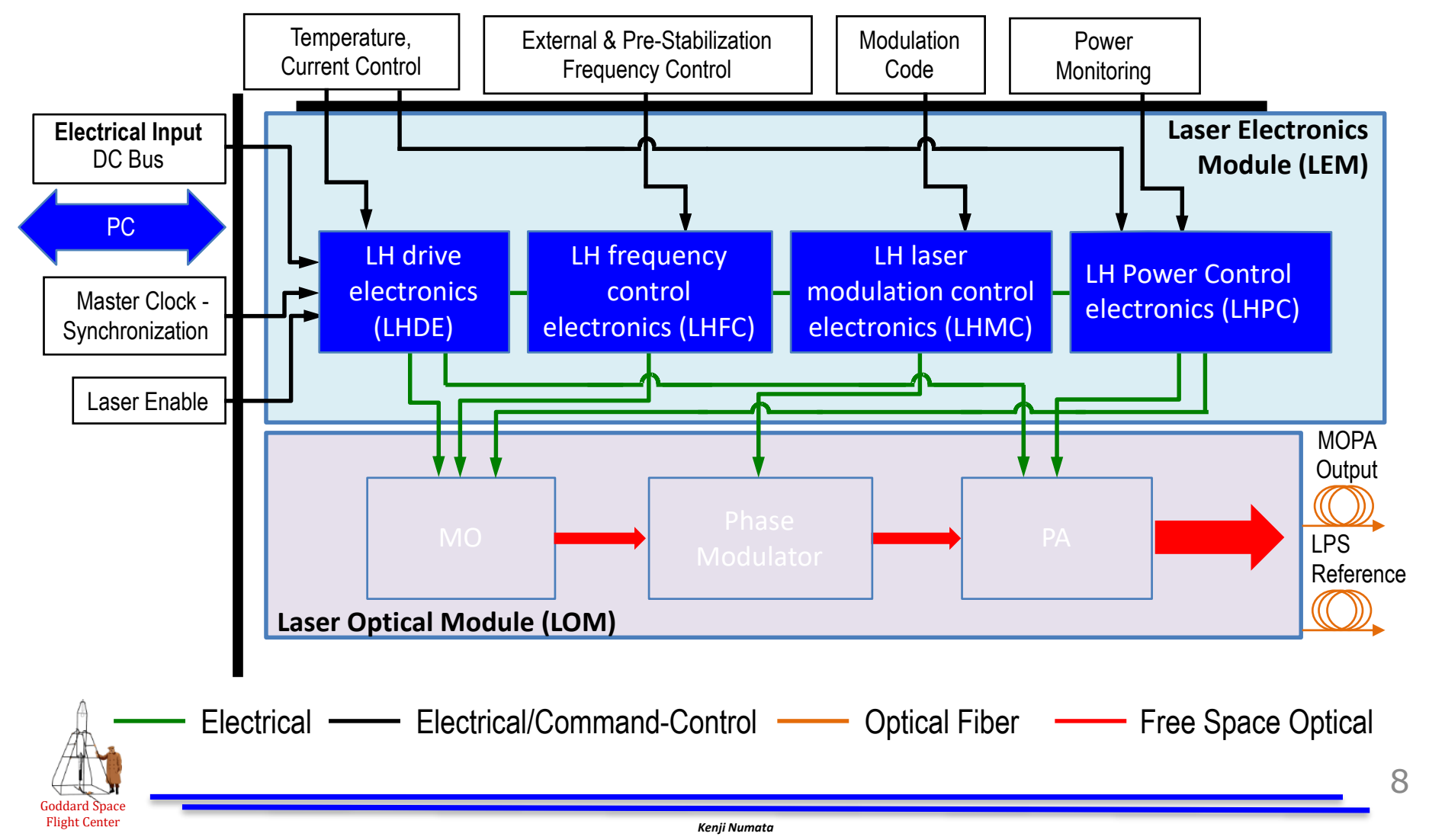


\section{NASA Laser Activities}

o To develop and improve the technology readiness level (TRL) to 6:

\begin{tabular}{|l|c|l|}
\hline \multicolumn{1}{|c|}{ Tasks } & GSFC Milestones & \multicolumn{1}{c|}{ ESA Milestones and Need Dates } \\
\hline $\begin{array}{l}\text { TRL4/5 Laser System Engineering Model (EM) } \\
\text { (no-Prestabilization) Delivery }\end{array}$ & $10 / 2019$ & $\begin{array}{l}\text { MFR (End Phase A): } \\
\text { November/December 2019 }\end{array}$ \\
\hline $\begin{array}{l}\text { TRL6 Laser System Engineering Test Unit } \\
\text { (ETU) with Pre-stabilization Delivery }\end{array}$ & $7 / 2021$ & $\begin{array}{l}\text { Unit-Level TRL 5/6 for Payload } \\
\text { Demonstrator items: end 2021 }\end{array}$ \\
\hline TRL6 Laser System ETU Lifetest & $\begin{array}{l}\text { implement at various } \\
\text { development stages }\end{array}$ & $\begin{array}{l}\text { "integrated-level" TRL 6 for adoption: } \\
\text { end 2023 }\end{array}$ \\
\hline
\end{tabular}

○ Significance of Work:

- Develop enabling technology for the LISA mission

- US contribution to the LISA mission led by ESA

- Approach:

- MOPA laser architecture that is consistent with the ESA approach for LISA

- Develop two MO approaches for risk mitigation

- Similar parallel approach for the PA development

- Integrate MO and PA to demonstrate MOPA performance meeting LISA's requirement.

- Develop and implement reliability test plan on critical components and systems. 


\section{MO approach 1 - GSFC micro NPRO}

- Conducted intensive research on different MO types

- Dropped fiber/semiconductor lasers because of their high intensity/phase noise

- Realized a smaller NPRO (non planar ring oscillator) crystal is more advantageous for LISA

o micro-NPRO features

- Larger free spectral range (FSR)

- Less chance of mode-hop, easier frequency overlapping between 6 lasers

- Better spatial overlap between pump and signal

- Minimized un-pumped region and optical loss

- Single spatial mode pumping for lowest phase noise

- Smaller package

- Use of telecom / semiconductor laser packaging technology

- Can fit more MOs into the allocated volume

○ Redesigned small, alignment-tolerant crystal

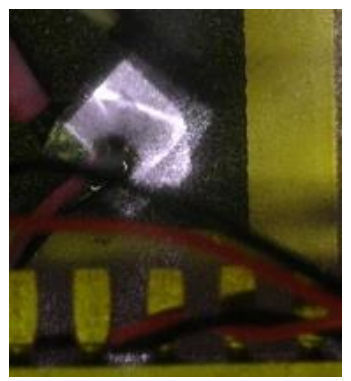

- $3 \mathrm{~mm}$ width, $~ 400 \mathrm{~mW}$ output max (in free-space, high enough for PA seeding)

- Goals are to reduce size and weight with improved performance, and to add extra redundancies and robustness. 


\section{NASA mNPRO breadboard performance}
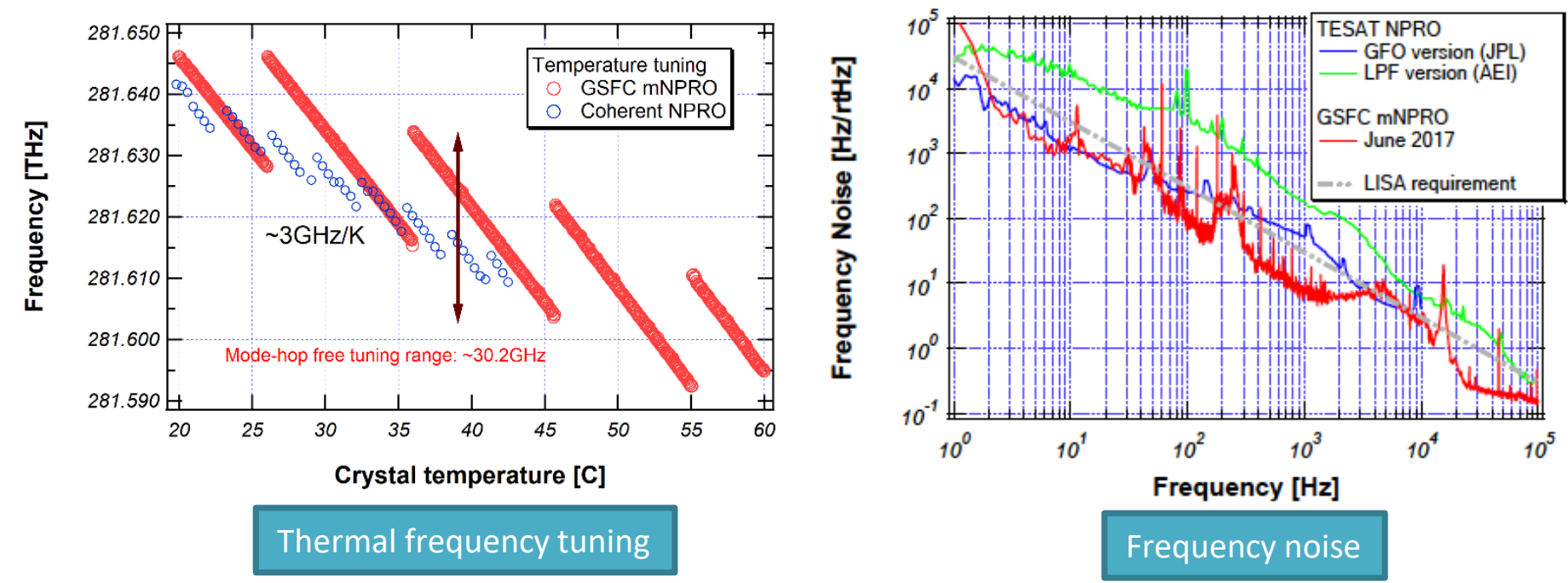

- Thermal frequency tuning coefficient

- Basically independent of crystal size

- Including $\mathrm{dn} / \mathrm{dT}$ and linear thermal expansion $\alpha$ for YAG

(7.3 $\times 10^{-6} / \mathrm{K}$ and $6.9 \times 10^{-6} / \mathrm{K}$, respectively)

- $\frac{d v}{d T}=-v(1 / n * d n / d T+\alpha)$

- Therefore, $\frac{\Delta v}{\Delta T} \approx-3.1 \frac{\mathrm{GHz}}{\mathrm{K}}$, agreeing with our measurement. 


\section{Frequency \& phase locking of mNPRO}

○ mNPRO has been frequency-locked to optical cavity, and phase-locked.

- 5kHz UGF with simple servo

- PZT resonance at $500 \mathrm{kHz}$ (with $3 \times 5 \times 0.5 \mathrm{~mm}$ PZT)

- Long-term locking with PZT + thermal servo loops
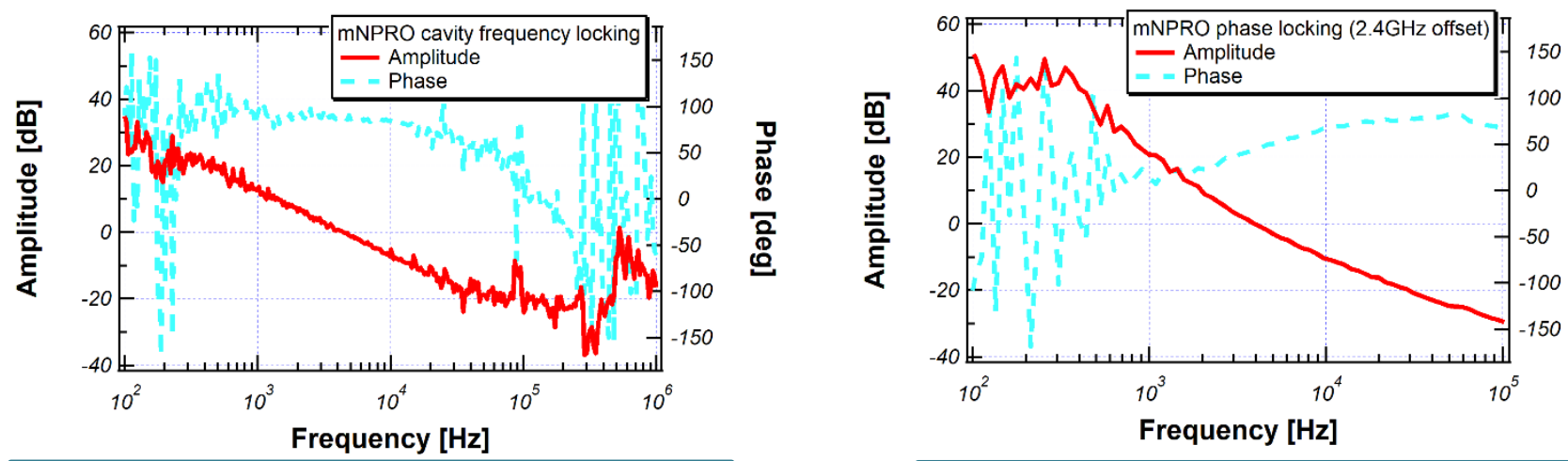

Open-loop transfer function of cavity frequency locking

Open-loop transfer function of offset phase locking

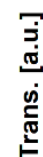

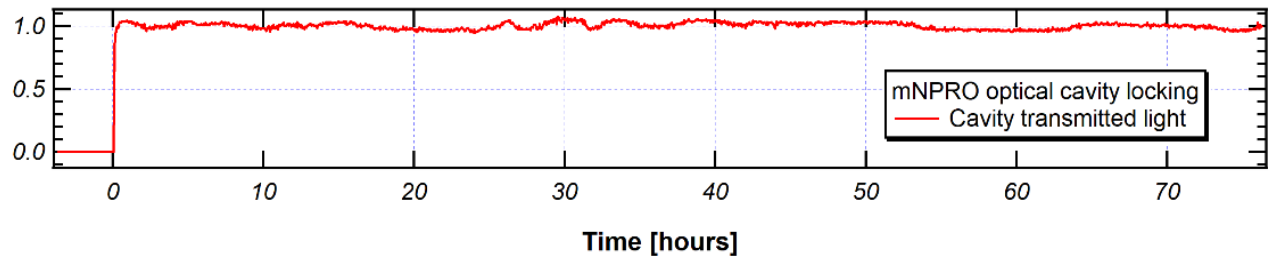

Long term cavity locking demo 
- Completed Pre-Design Review (PDR) - 3/2018

○ $1^{\text {st }}$ package delivery: $8 / 2018$

- A mechanical model has been developed in accordance with optical design and required optical alignment tolerances.

- The design allows for minimal mechanical changes to move from prototype versions where epoxy is predominantly used to hold/align optics to production where epoxy is eliminated in favor or welded or soldered components.

- The design has been developed to be modular in nature such that key subassemblies may be assembled, characterized, and qualified outside the overall system.

All materials and processes are under control of NASA. Looking for flight opportunities before LISA.
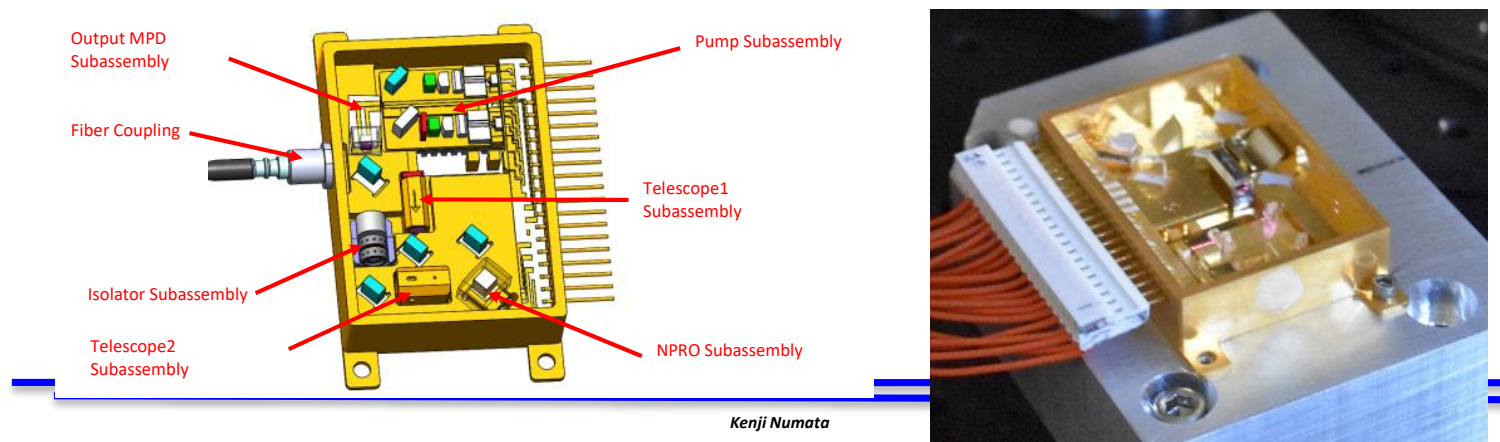
- GSFC in-house fiber amplifier

- >2W, continuous-wave output

- All-fiber configuration

- Double-clad large mode area (LMA) fiber

- Forward pumping for safety \& lower SBS noise
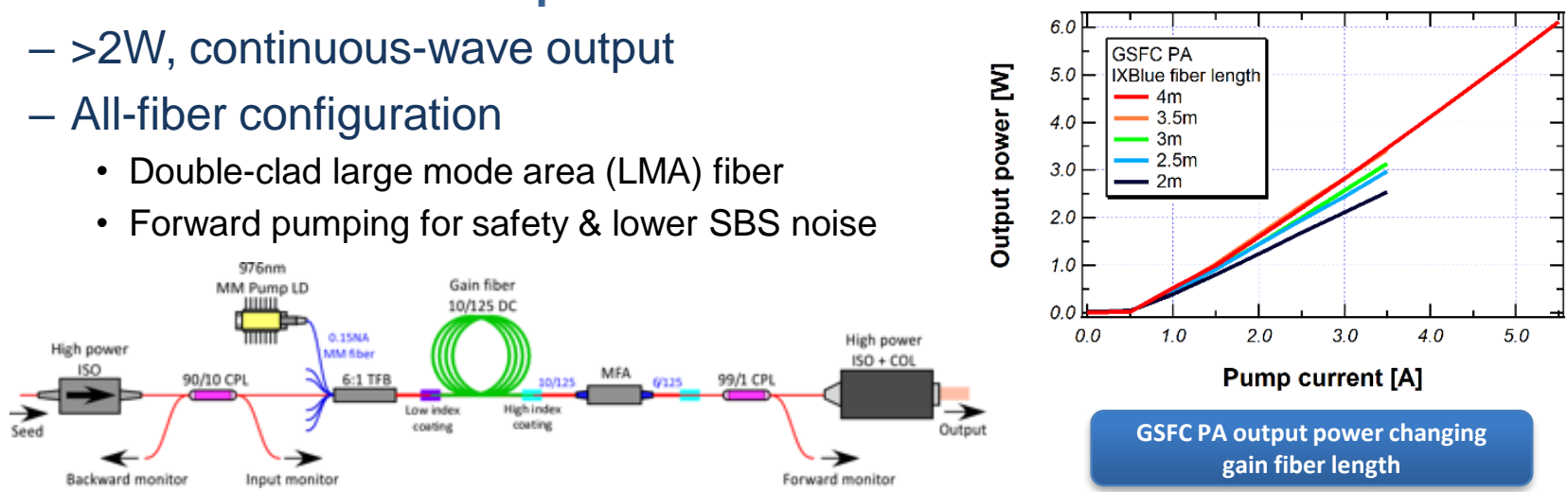

GSFC PA output power changing gain fiber length

- External vendor amplifiers

- Fibertek \& Advalue: delivering to GSFC
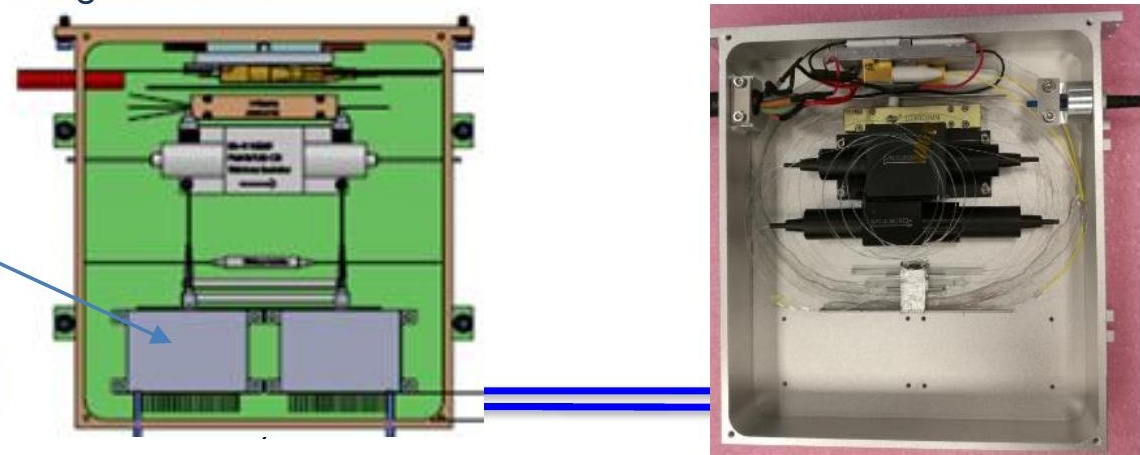


\section{NASA GSFC MO + PA brassboard}

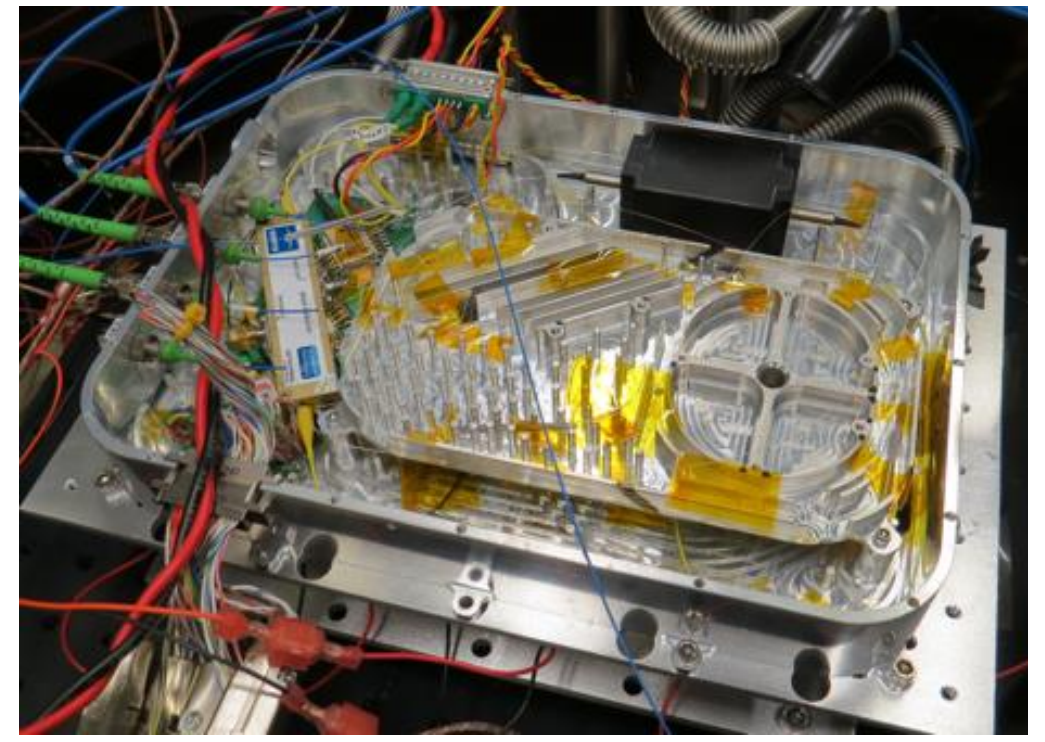

- Test amplifier built by GSFC in TVAC chamber.

- Used for environmental testing and intensity stabilization demonstration.

- Current Dimensions- 260 mm (L) x $190 \mathrm{~mm}(\mathrm{~W}) \times 40 \mathrm{~mm}(\mathrm{H})$

- This package includes both semiconductor seed laser and a fiber amplifier pumped with a single pump diode.

- We plan to repackage this fiber amplifier to fit within the $\mathrm{LH}$ volume

- Have done early qualification tests (vib, TVAC, radiation, etc.)

- Starting low-level life testing on pump diodes (for MO and PA) 


\section{NASA MO + PA system noise}

- System noise demonstrated to satisfy LISA requirements

- Master oscillator + power amplifier

- Will be revisited with packaged m-NPRO, new PAs, and cavities.

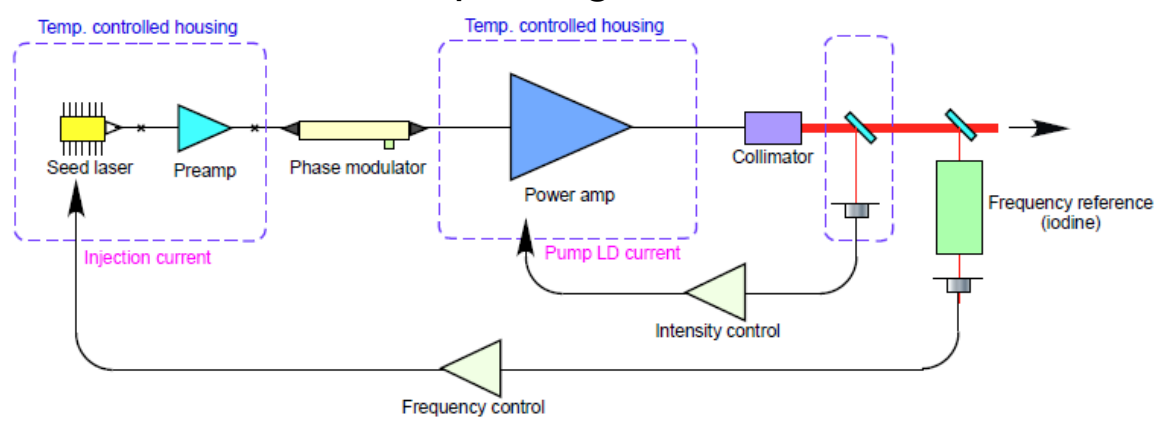

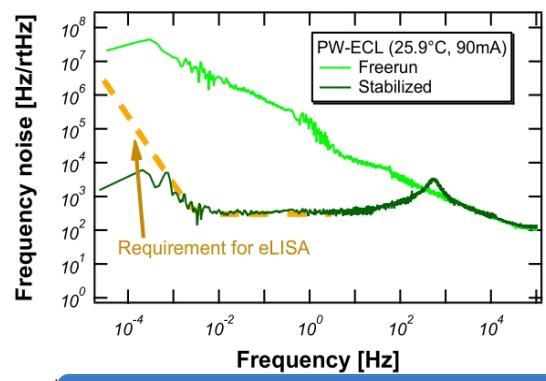

Stabilized frequency noise of PW-ECL + GSFC PA

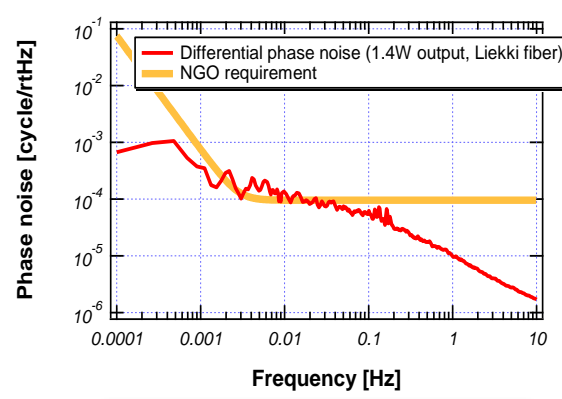

Differential phase noise of GSFC PA

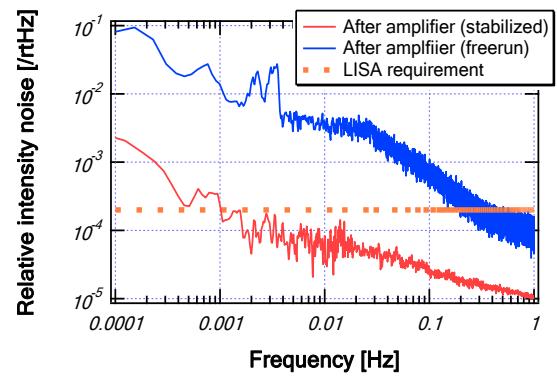

Stabilized intensity noise of GSFC PA 


\section{Risk assessment}

- Reliability / lifetime will be an important issue.

- Components (vendors) availability is not trivial for the 2030's launch.

\begin{tabular}{|c|c|c|c|}
\hline Risks & Likelihood & Mitigation & $\begin{array}{l}\text { Post- } \\
\text { Mitigation } \\
\text { Residual } \\
\text { Likelihood }\end{array}$ \\
\hline Reliability / lifetime & Medium to High & $\begin{array}{ll}\text { - } & \text { Derating } \\
\text { - } & \text { Life test } \\
\text { - } & \text { Leverage lessons learned from previous missions } \\
\text { - } & \text { Redundancy } \\
& \text { Components evaluation and selection }\end{array}$ & Medium \\
\hline $\begin{array}{l}\text { Early failure of pump } \\
\text { diodes }\end{array}$ & Low to Medium & $\begin{array}{l}\text { - } \quad \text { CW pump diodes with derating } \\
\text { - } \quad \text { Lower peak power than previous missions }\end{array}$ & Low \\
\hline Laser damage & Low to Medium & $\begin{array}{l}\text { - Internal fluence significantly lower than previously built lasers } \\
\text { - } \quad \text { Pressurized enclosure to minimize contamination induced damage } \\
\text { - } \quad \text { Follow established quality control and build processes }\end{array}$ & Low \\
\hline $\begin{array}{l}\text { Laser system doesn't meet } \\
\text { LISA noise requirements } \\
\text { after environmental } \\
\text { testing }\end{array}$ & Low & $\begin{array}{l}\text { - } \quad \text { Build engineering model using knowledge gained from laboratory studies. } \\
\text { - Maintain vigorous testing program throughout laser program }\end{array}$ & Low \\
\hline $\begin{array}{l}\text { Components/Vendors } \\
\text { Availability }\end{array}$ & Medium to High & $\begin{array}{ll}\text { - } & \text { Qualify multiple vendors as early as possible } \\
\text { - } & \text { Reliability tests } \\
\text { Work closely with vendors to address issues and find replacement }\end{array}$ & Medium \\
\hline$\underset{\text { Goddard Space }}{\text { Flight Center }}=$ & & & 17 \\
\hline
\end{tabular}




\section{NASA Pump laser diode testing facility at GSFC}

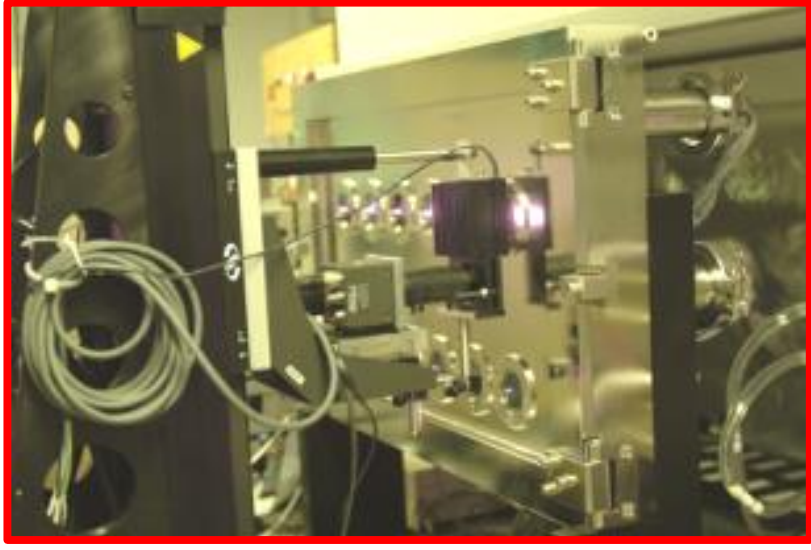

Fully automated custom vacuum chamber with 12 LDA test positions \& windows for continuous inspection

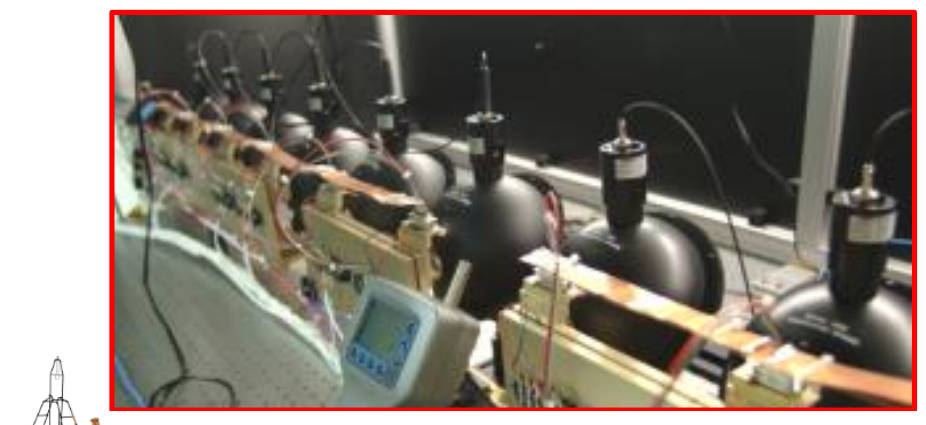

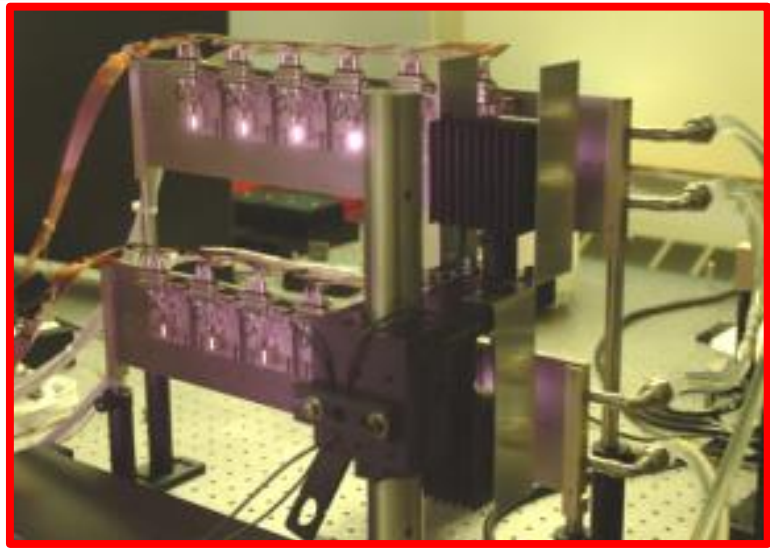

Fully automated custom test station with 12 LDA test positions \& continuous power measurement capability

$2^{\text {nd }}$ test station with 12 LDA test positions \&

continuous power measurement capability

- Have procured many pump LDs for the MO \& PA to start testing 
Three designated rooms for space flight lasers build

○ Room \#1

- Bonded storage of flight hardware.

- Wet and dry cleaning stations for flight hardware

- Room \#2

- 3 optical tables for the flight build as well as storage of any hardware \& tools needed during the building process.

o Room \#3

- TVAC chamber for TVAC cycling.

- Optical table for testing during TVAC cycling.

- Currently SLAC is used by GEDI (launch next month).

- LISA will be the next user of SLAC (if funded).
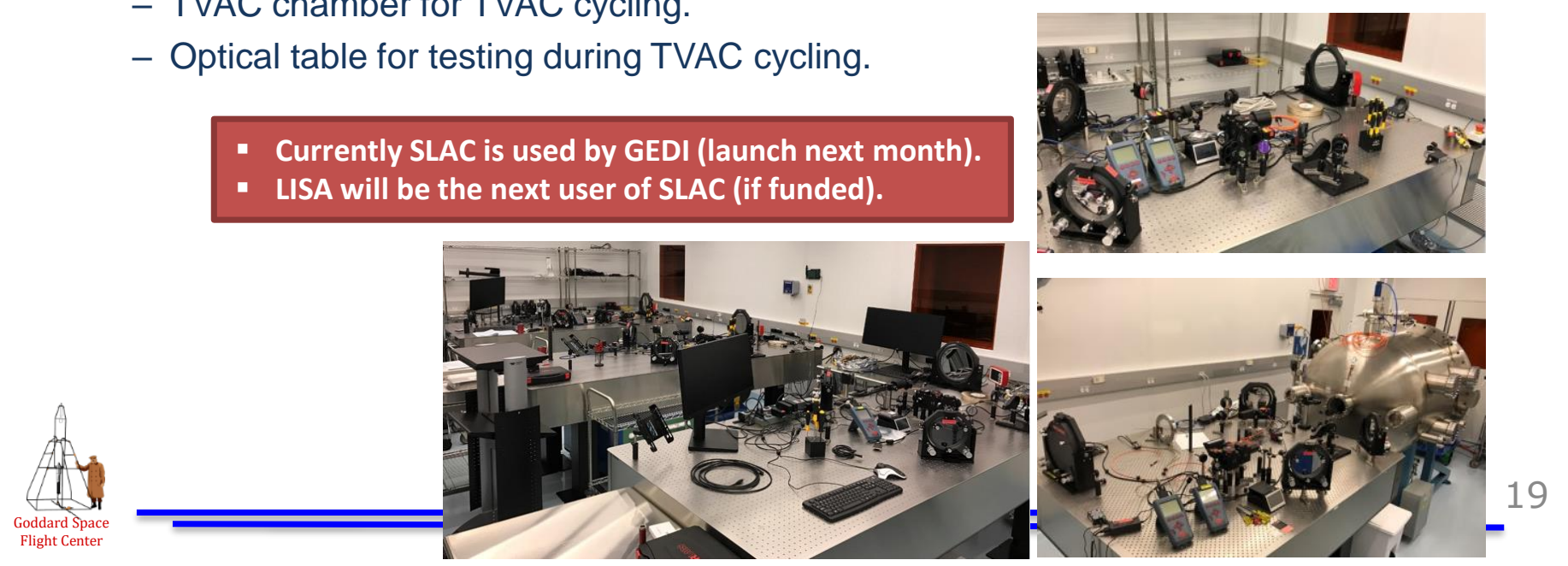


\section{Summary}

- We are developing LISA laser paying attentions to:

- Noise

- Requirements achieved at breadboard level

- PA selection primarily dependent on RF phase noise.

- Will revisit noise performance with packaged MO and PA.

- Lifetime/reliability

- Minimizing risks by design, test, and analysis

- Establishing test plans

- Awaiting for funding to start full reliability activities

- We are currently on track to meet the TRL6 plan for the MOPA laser.

- TRL4/5 Laser System Engineering Model (EM) delivery: 10/2019

- TRL6 Laser System Engineering Test Unit (ETU) delivery: 7/2021

- We plan to transfer technologies to the industry for the flight lasers build

- We will work with the selected vendor closely to transfer necessary technologies for building the flight lasers.

- We will perform acceptance testing on all deliverables prior to delivery to ESA. 